\title{
ESPECTROSCOPIA CEREBRAL EM CANDIDATOS A TRANSPLANTE HEPÁTICO
}

\author{
Gustavo Justo Schulz*1, Julio Cézar Uili Coelho', Jorge Eduardo fouto Matias', Antônio Carlos ligocki Campos', Danielle Duck Schulz', \\ Guilherme Augusto Bertoldi ${ }^{1}$
}

Trabalho realizado no Serviço de Transplante Hepático do Hospital de Clínicas da Universidade Federal do Paraná, Curitiba, PR

\author{
*Correspondência: \\ Rua Professor Pedro Viriato \\ ParigotdeSouza, 1900 apto 604 \\ bloco 6 \\ CEP 81200-100 - Curitiba-PR \\ e-mail:gjschulz@hotmail.com
}

\begin{abstract}
RESUMO
OBJEtivos. Determinar os níveis dos metabólitos (mio-inositol [MI], colina [Cho], glutamina [G|X], creatina [Cr] e N-acetilaspartato [NAA]) por meio da espectroscopia por ressonância magnética em portadores de hepatopatia crônica, antes e após o transplante hepático, correlacionando com a avaliação clínica.

Métodos. Foram estudados prospectivamente 25 pacientes portadores de hepatopatia crônica do Serviço de Transplante Hepático da Universidade Federal do Paraná por meio de avaliação clínica e espectroscópica. Trinta voluntários sadios formaram o grupo controle, sendo submetidos às mesmas avaliações. Dezesseis dos 25 pacientes também foram avaliados após o transplante.

REsultados. Antes do transplante hepático reduções significativas nos índices de MI/Cre Cho/Cre aumento significativo no índice de Glx/Cr foram observadas nos pacientes portadores de encefalopatia hepática comparados ao grupo controle. Os critérios quantitativos de Ross para diagnóstico espectroscópico da encefalopatia hepática (MI//Cr e Cho/ $\mathrm{Cr}<$ média +2 desvios padrão do grupo controle) demonstraram uma sensibilidade de $61,54 \%$, especificidade de $91,67 \%$ e precisão de $76 \%$, sendo que a Cho/Cr foi o melhor parâmetro isolado. A espectroscopia após o transplante mostrou mudanças nos índices metabólicos comparados com o status pré-transplante.
\end{abstract}

Conclusão. A espectroscopia permite um diagnóstico preciso da encefalopatia hepática. A melhora dos níveis metabólicos após o transplante hepático sugere um importante papel do Ml e da Cho no desenvolvimento da encefalopatia hepática.

Unitermos: Análise espectral. Encefalopatia. Transplante de figado. Espectroscopia de ressonância magnética.

\section{INTRODUÇÃO}

A encefalopatia hepática $(E H)$ é uma disfunção neuropsiquiátrica reversível, secundária à insuficiência hepátical, ,cuja prevalência é variável. É comumente subestimada em virtude da preservação das habilidades verbais dos pacientes em estágios iniciais da doença. Os testes neuropsicométricos são considerados padrão-ouro no diagnóstico da encefalopatia hepática, porém a sua utilização isolada pode ocasionar erros de interpretação, já que não são específicos dessa doença, além de sofrerem influências da idade e nível de instrução dos pacientes e revelarem pouco sobre o processo neuroquímico envolvido na encefalopatia ${ }^{3}$.

Evidências crescentes na literatura apontam a importância do diagnóstico e da instituição precoce do tratamento da encefalopatia, de preferência antes do aparecimento de sinais clínicos, visando à preservação das funções cerebrais. Recentemente, refinamentos técnicos na espectroscopia por ressonância magnética (RM) tornaram possível o estudo bioquímico do cérebro humano in vivo, com a identificaçãa e quantificaçãa dos metabólitos cerebrais em regiões espećfícas4,8,22,29. A encefalopatia hepática é caracterizada por depleção do mio-inositol (MI) e da colina (Cho) associada a um aumento do complexo glutamino-glutamato (Glx). Estas alterações não são encontradas em outras encefalopatias ${ }^{4,5,28}$.
O transplante hepático é o único tratamento capaz de reverter as disfunções neurológicas verificadas na encefalopatia hepática, evitando o desenvolvimento da degeneração hepatocerebral. A reversibilidade da encefalopatia hepática com o transplante pode ser demonstrada, bioquimicamente, pela espectroscopia, ${ }^{3,4,6}$. O objetivo do presente estudo é determinar as alterações dos níveis dos metabólitos cerebrais por meio da espectroscopia em candidatos a transplante hepático, antes e após o procedimento cirúrgico.

\section{MÉtodos}

Entre outubro de 2002 e novembro de 2003, realizou-se um estudo prospectivo com portadores de doença hepática crônica, candidatos a transplante no Serviço de Transplante Hepático do Hospital de Clínicas da Universidade Federal do Paraná. Os pacientes foram avaliados por meio de testes neuropsicométricos e espectroscopia.

O grupo de estudo foi formado por 25 pacientes, sendo 19 (76\%) do sexo masculino e seis (24\%) do sexo feminino. A média de idade foi de $5 I, I \pm 10,27$ anos (variando de 31 a 70 anos). A principal causa de disfunção hepática foi hepatite C (44\%). A maioria dos pacientes (72\%) eram Child-Pugh B. 
O grupo controle foi constituído por 30 voluntários sadios. A média de idade do grupo controle foi de 36,43 $\pm 12,41$ anos (variando de 14 a $6 \mathrm{I}$ anos). Dezesseis (53,33\%) eram do sexo masculino e I 4 (46,67\%) do sexo feminino.

A encefalopatia hepática foi diagnosticada observando-se os dados do exame clínico, exame neurológico e dos testes neuropsicométricos (TNPS). Para graduação da encefalopatia hepática clínica utilizou-se os critérios de Parsons-Smith7 e para a disfunção hepática, a classificaçãa de Child e Pugh. A avaliação das funções cognitivas foi realizada por meio de TNPS, que incluíram: miniexame do estado menta ${ }^{8,9}$, teste de trilhas $A$ e $B^{10}$, subteste de dígitos da bateria Wais- $R^{\prime \prime}$, teste de fluência verbal (FAR) e categorias ${ }^{10}$ e teste do desenho do relógio ${ }^{10}$.

Para fins de comparação, os indivíduos avaliados foram divididos em três grupos distintos, de acordo com exame clínico e TNPS:

- Grupo I (GRI): pacientes com EH $(n=13)$;

- Grupo 2 (GR2): pacientes sem EH $(n=12)$;

- Grupo 3 (GRC): grupo controle - voluntários sadios $(n=30)$

Dezesseis pacientes do grupo de estudo foram submetidos a transplante hepático e às mesmas avaliações (clínicas e espectroscópicas) no primeiro mês de pós-operatório. Destes, 14 puderam ser reavaliados com 90 dias de pós-operatório.

A espectroscopia foi realizada com um aparelho de ressonância com I,5T (Magnetom Vision; Siemens, Erlanger, Germany) utilizandose uma bobina polarizada circular posicionada junto à cabeça dos examinados. Para os exames de controle pós-operatório, a exata reprodução da localização espectral foi conseguida mediante a aquisição prévia de três cortes (sagital, transversal e coronal) na RM em T2 (tempo de relaxamento transversal dos núcleos) utilizando-se a técnica de fast spin echo para posicionamento tridimensional do volume de interesse (voxe) na parte medial do lobo occipital (substância branca e cinzenta) com $2 \times 3 \times 2 \mathrm{~cm}^{8,12}$

Para detecção e quantificação do Ml e Glx, foram utilizadas sequências espectroscópicas com tempo de eco (TE) curto, ou seja, $20 \mathrm{mseg}$. O tempo de repetição (TR) escolhido foi de $1500 \mathrm{mseg}$ associado a uma supressão do sinal dominante da água ${ }^{18}$. Para sequência de pulso, optou-se pela técnica PRESS ("point-resolved spectroscopy"). A análise da curva espectral foi feita por meio de um software espećífico desenvolvido pela GE/Siemens, mediante o qual se consegue localizar os picos de cada metabólito conforme a frequência espectral correspondente ${ }^{3,13-19}$.

Em virtude da intensa sobreposição de Glx com o pico dos radicais metileno (amino-butirato, NAA e aspartato), uma integração manual foi processada entre 2, I 5 e 2,45ppm para o pico de Glx, com o objetivo de representar fielmente 0 nível de glutamina ${ }^{18}$. Este foi o único metabólito em que se avaliou a área envolvida abaixo da curva e não o pico, tendo em vista não formar um pico único como os outros metabólitos analisados.

Após a determinação de cada pico a ser estudado e seus respectivos valores em Unidades de Ressonância (UR), fornecidos pelo software da GE/Siemens, foram calculados os índices absolutos para MI, Cho e Glx em relação a $\mathrm{Cr}$, usada como padrão de comparação, conforme proposto por Kreis, em 1992, ${ }^{4}$ e Ross, em 19943. O grupo controle foi usado como referência da normalidade em função da técnica de RM utilizada neste estudo.
O diagnóstico espectroscópico da encefalopatia hepática foi estabelecido segundo os Critérios de Ross ${ }^{3}$, ou seja, quando os pacientes apresentavam o $\mathrm{Ml} / \mathrm{Cr}$ e $\mathrm{Cho} / \mathrm{Cr}$ dois desvios padrão abaixo dos controles ( $\mathrm{MI} / \mathrm{Cr}$ e $\mathrm{Cho} / \mathrm{Cr}<$ média -2 desvios padrão do grupo controle) com ou sem $\mathrm{Glx} / \mathrm{Cr}$ um desvio padrão acima dos controles ( $\mathrm{Gl} / \mathrm{C} / \mathrm{r}>$ média + I desvio padrão do grupo controle). Esses critérios foram comparados com o teste padrão (TNPS) para avaliação dos índices de qualidade da espectroscopia no diagnóstico da encefalopatia hepática. Repetiram-se os critérios em cada reavaliação dos pacientes transplantados com 30 e 90 dias da cirurgia. Considerou-se como melhora clínica no pós-transplante a normalização de pelo menos um dos TNPS alterados no pré-transplante.

Foram testadas as diferenças significativas entre os grupos por meio do teste não-paramétrico de Kruskal-Wallis. Aplicaram-se os mesmos critérios em cada reavaliação.

\section{Resultados}

\section{Antes do transplante hepático}

Dos 25 pacientes avaliados, 13 apresentavam encefalopatia hepática (EH), sendo clinicamente aparente em apenas um paciente (4\%).

O índice médio de NAA/Cr nos pacientes do GRI (com EH) foi $1,92 \pm 0,24$, não apresentando diferença estatística em relação aos pacientes do GR2 (sem EH) e GRC, cujos índices foram $1,91 \pm 0,22$ e I,89 $\pm 0,28$, respectivamente (teste de Kruskal-Wallis, $p=0,9 \mid 26$ ). Em relação ao $\mathrm{MI} / \mathrm{Cr}$, o índice médio nos pacientes do GRI foi $0,49 \pm 0,10$ e nos pacientes do GR2 foi $0,56 \pm 0,17$, sendo em ambos os grupos inferior a dois desvios padrão do índice médio do GRC, $0,83 \pm 0,13$ (teste de Kruskal-Wallis, $p<0,000$ I). Resultados similares foram observados para o índice médio de Cho/Cr: 0,54 $\pm 0,15$ nos

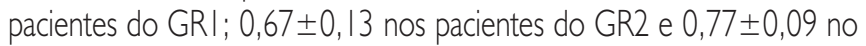
GRC (teste de Kruskal-Wallis, $p=0,000 \mathrm{I}$ ). A diferença no índice médio do $\mathrm{Glx} / \mathrm{Cr}$ entre os grupos foi menor, porém igualmente significativa: I, I2 $\pm 0,26$ no GRI; I,00 $\pm 0,28$ no GR2 e 0,89 $\pm 0,19$ no GRC (teste de Kruskal-Wallis, $p=0,0 \mid 46$ ).

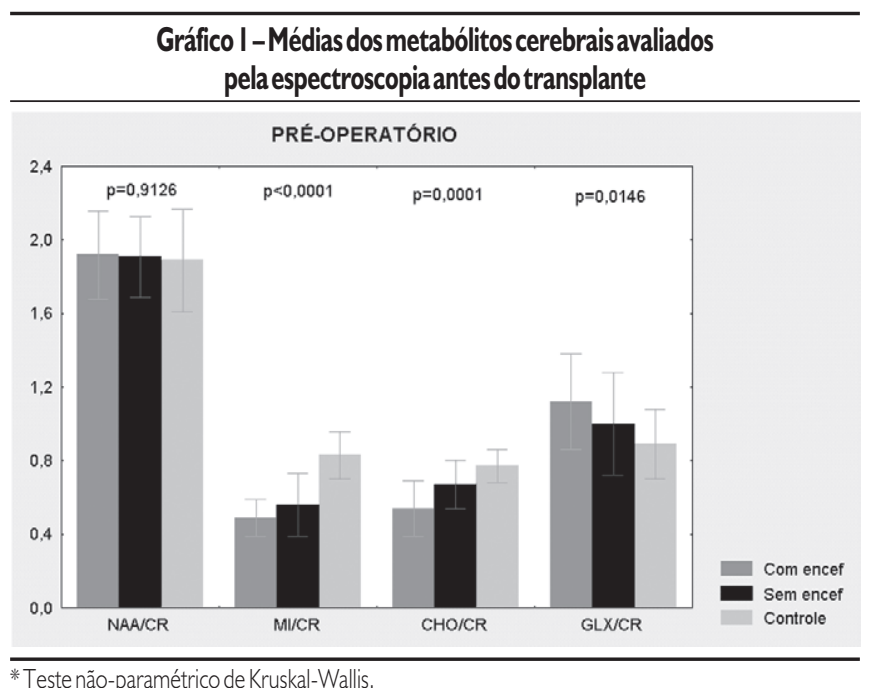

*Teste não-paramétrico de Kruskal-Wallis 
Em uma análise geral, o índice Ml/Cr encontrava-se depletado em 64\% dos pacientes, o índice Cho/ $\mathrm{Cr}$ em $48 \%$, enquanto a $\mathrm{Glx} / \mathrm{Cr}$ encontrava-se acima dos controles em $52 \%$ dos pacientes.

Dos 13 pacientes com EH pelos TNPS, a espectroscopia foi positiva em oito (61,5\%) e negativa em II dos 12 pacientes sem EH (91,6\%). Um paciente apresentava critérios espectroscópicos para diagnóstico de EH, apesar de não apresentar alterações nos TNPS. Cinco pacientes apresentavam espectroscopia falso-negativa, acarretando um valor preditivo negativo (VPN) baixo. Quando se utilizaram os critérios de Ross para diagnóstico espectroscópico da $\mathrm{EH}$, a sensibilidade (S) foi de 61,54\%, a especificidade (E) de $91,67 \%$, o valor preditivo positivo (VPP) de $88,89 \%$, o valor preditivo negativo (VPN) de 68,75\% e a precisão de 76\%.

Quando se analisou apenas a Cho/Cr como critério diagnóstico da EH na espectroscopia, observaram-se resultados mais parecidos aos obtidos com a utilização dos critérios de Ross, porém com maior sensibilidade e menor especificidade. Nesse caso, a sensibilidade foi de $76,92 \%$, especificidade de $83,33 \%$ e precisão de $80 \%$.

Dos 16 pacientes transplantados com seguimento pós-operatório, os índices de $\mathrm{MI} / \mathrm{Cr}$, Cho/ $\mathrm{Cr}$ e $\mathrm{Gl} / \mathrm{Cr}$ encontravam-se em níveis patológicos em seis pacientes. O exame clínico e os TNPS revelaram sinais de EH em cinco dos seis pacientes. Os cinco pacientes preenchiam os critérios espectroscópicos para diagnóstico de EH definidos por Ross et al $\left.\right|^{3}$.

\section{Após o transplante hepático}

\section{Avaliação em 30 dias de pós-operatório}

Houve acréscimo dos valores de $\mathrm{MI} / \mathrm{Cr}$ e $\mathrm{Cho} / \mathrm{Cr}$ e decréscimo dos valores de $\mathrm{Gl} / \mathrm{C} / \mathrm{Cr}$, não existindo mais diferença estatística em relação ao grupo controle. Valores patológicos de $\mathrm{Glx} / \mathrm{Cr}$ ainda persistiam em alguns pacientes. Não houve diferença estatística em relação aos índices de NAA/Cr com 30 dias de pós-operatório.

\section{Avaliação em 90 dias de pós-operatório}

A avaliação envolveu nove pacientes do grupo com EH e cinco pacientes do grupo sem EH.

Houve melhora clínica (exame clínico e TNPS) em sete dos nove pacientes do grupo com EH; e nenhum deles, à avaliação espectroscópica cerebral, preenchia os critérios de Ross para diagnóstico de $\mathrm{EH}$. Dos dois pacientes que não obtiveram melhora clínica, o $\mathrm{MI} / \mathrm{Cr}$ encontrava-se em níveis patológicos em um deles.

Ao analisar-se a espectroscopia isoladamente, nota-se um acréscimo de $\mathrm{MI} / \mathrm{Cr}$ e $\mathrm{Cho} / \mathrm{Cr}$ acima dos controles em ambos os grupos. $\mathrm{O}$ índice de Glx/Cr praticamente equiparou-se aos controles. Não houve diferença estatística em relação ao índice de NAA/Cr.

\section{Comparação dos índices espectroscópicos nos diferentes momentos da avaliação}

Para o MI/Cr, percebeu-se uma melhora significativa $(p<0,05)$ nos valores absolutos após 30 dias da realização do transplante hepático, chegando a suplantar os controles com 90 dias de pós-operatório em ambos os grupos.

$\mathrm{Na}$ avaliação do índice de $\mathrm{Cho} / \mathrm{Cr}$, verificou-se um acréscimo nos valores absolutos, suplantando os controles com 30 e 90 dias de

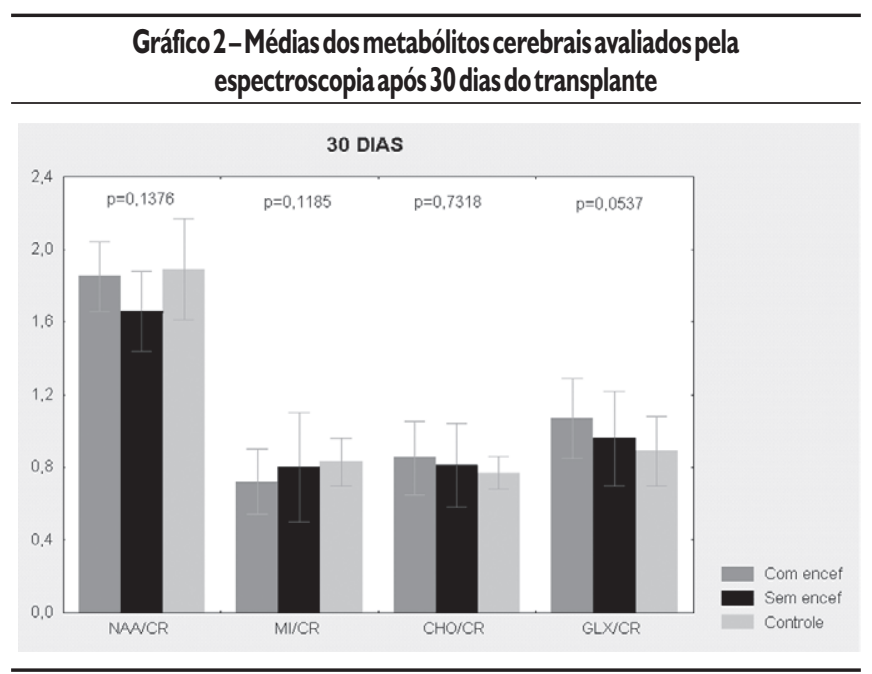

* Teste não-paramétrico de Kruskal-Wallis

** Há um acréscimo de Cho/Cracima dos controles para ambos os grupos

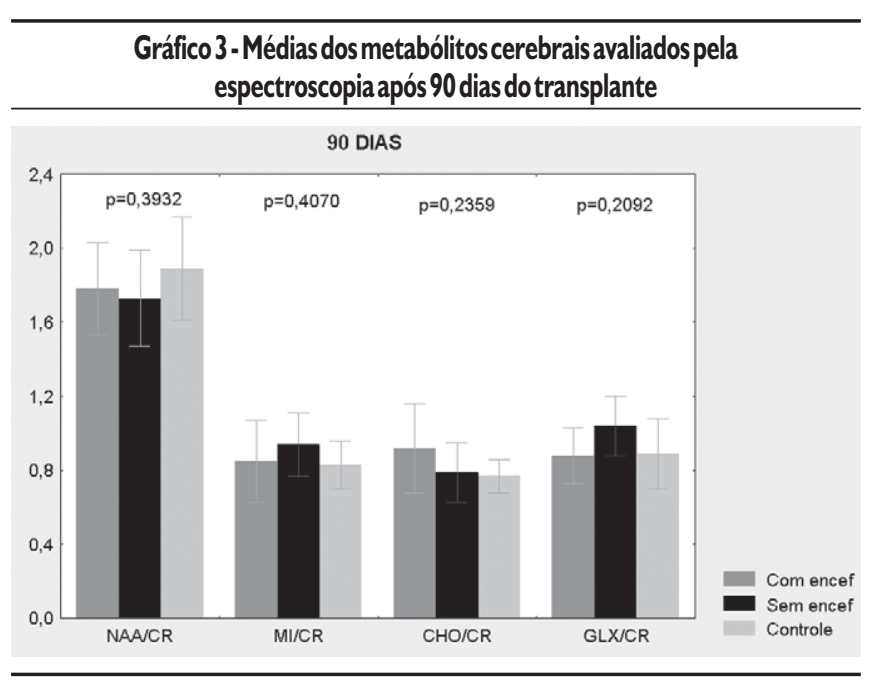

* Teste não-paramétrico de Kruskal-Wallis.

pós-operatório. Para esta variável a diferença não foi significativa no grupo sem EH em nenhum momento.

Quanto ao índice de $\mathrm{G} / x / \mathrm{Cr}$, houve um decréscimo mais tardio, ou seja, após 90 dias de pós-operatório para os pacientes com EH. Portanto, com a utilização dos critérios de Ross ou dos índices MI/Cr e $\mathrm{Cho} / \mathrm{Cr}$ isoladamente atingiu-se $100 \%$ de especificidade.

$\mathrm{O}$ índice que atingiu a maior sensibilidade para diagnóstico da reversão da EH foi o MI/Cr, com 50\%, além de um VPP de I00\%, VPN de $87,5 \%$ e precisão de $88,89 \%$.

\section{Discussão}

No Brasil, o número de transplantes aumentou expressivamente a partir da década de $90 \mathrm{com}$ a nova lei de transplante de órgãos e tecidos, quando o Sistema Único de Saúde (SUS) passou a pagar os 
hospitais credenciados. No serviço de transplante da UFPR são realizados em média 35 transplantes hepáticos por ano.

No período pós-transplante, as alterações neurológicas são causas frequentes de morbimortalidade, podendo afetar 13,2\% a $90 \%$ dos pacientes, conforme diferentes séries ${ }^{20}$.

Estudos utilizando TNPS, EEG e espectroscopia têm demonstrado que disfunções cognitivas (EH leve ou subclínica) são comuns em pacientes portadores de hepatopatia crônica com função mental aparentemente normal ao exame clínico, 5, 15,22-25.

A prevalência de EH subclínica nesta casuística foi de $48 \%$. Este dado coincide com a literatura, que aponta uma prevalência de EH subclínica em portadores de cirrose hepática que varia de 44\% a 100\% dos casos, dependendo dos testes utilizados e da população estudada 3,23,24,26. As funções sabidamente afetadas nos estágios precoces da EH são as psicomotoras e funções visuo-espaciais, ambas de difícil detecção no exame neurológico habitual e, frequentemente, negligenciadas sem a utilização de testes específicos ${ }^{3}$. A importância da EH subclíica pode ser demonstrada por meio de três fatores principais. Primeiro, esses déficits neuropsicométricos podem resultar em redução da habilidade dos indivíduos em executar tarefas simples do dia-a-dia, conferindo um risco aumentado de acidentes de trabalho. Segundo, podem se traduzir em deterioração da qualidade de vida destes pacientes. Terceiro, a EH subclínica poderia ser um marcador de futuros episódios de EH clinicamente aparente, sendo que muitos estudos demonstram disfunções cerebrais progressivas em pacientes que não apresentavam evidências clínicas de EH ${ }^{25}$. Nesta casuística, $52 \%$ dos pacientes avaliados apresentaram pelo menos dois TNPS alterados.

A história natural da EH subclínica ainda não é bem conhecida. Em um artigo bastante provocativo, Krieger ${ }^{16}$ demonstrou a presença de déficits neurológicos persistentes a despeito do tratamento clínico da EH em cirróticos. Baseado nestes achados, sugeriu que a EH poderia ser uma desordem neurodegenerativa lentamente progressiva ${ }^{16,32}$. Isto vai contra o fato de a EH ser encarada como uma simples encefalopatia metabólica reversível. Contudo, o transplante hepático parece reverter as anormalidades motoras e cognitivas dos cirróticos, como demonstrado no presente estudo, e danos estruturais maiores no cérebro são incomuns. Mais estudos são necessários para confirmar a aparente resolução das disfunções corticais e subcorticais nos pacientes com EH submetidos a transplante.

As alterações metabólicas cerebrais encontradas nos cirróticos com EH clínica ou subclínica podem ser detectadas de maneira nãoinvasiva com a utilização da espectroscopia. Este exame apresenta especificidade próxima a 100\%, uma vez que as alterações identificadas na EH (depleção do MI/Cre e Cho/Cr com ou sem alteração do $G \mid x)$ não são encontradas em outras encefalopatias ${ }^{21}$. $O$ que se defende é a utilização conjunta da espectroscopia e TNPS, o que determinaria uma alta sensibilidade (TNPS) associada a uma alta especificidade (espectroscopia) no diagnóstico da EH. Isto é particularmente importante no período pós-transplante, em que vários fatores podem ser causa de alterações neurológicas nos transplantados e de difícil discernimento clínico entre eles.

Poucos estudos na literatura descrevem os achados espectroscópicos em pacientes cirróticos submetidos a

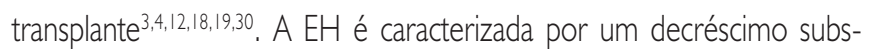
tancial no mio-inositol (MI) e colina (Cho) associado a um aumento tardio de glutamina (Glx).

A despeito dos achados espectroscópicos positivos, o papel do MI e da Cho no cérebro humano, e especialmente em EH, não está completamente esclarecido. Na disfunção hepática crônica, uma diminuição da atividade do ciclo da ureia parece ser a responsável pela elevação do nível cerebral da glutamina, cuja síntese é aumentada nos astrócitos $^{14}$. Tem sido demonstrado que o Ml, que está localizado quase que exclusivamente nos astrócitos, pode servir como um metabólito osmoticamente ativo e tem um importante papel na regulação do volume destas células ${ }^{5,14}$. É relatado que o Ml é considerado um marcador astrocitário, tanto quanto seu mensageiro neuronal polifosfato de inositol, admitindo-se um papel excitatório maior. Sendo assim, o edema das células da glia induzido pela amônia (não metabolizada pelo fígado cirrótico ou desviada por derivações portossistêmicas) induz uma alta concentração celular de glutamina e liberação compensatória de $\mathrm{Ml}$ desencadeando a $\mathrm{EH}$. Neste mesmo raciocínio, pode-se encaixar a Cho, que também é considerada um metabólito osmoticamente ativo presente na mielina, membranas celulares e outros complexos cerebrais lipídicos. Em condições patológicas pode sofrer alterações como ocorrem com o MI.

\section{CONCLUSÃo}

A espectroscopia permite um diagnóstico preciso da encefalopatia hepática, mesmo em seus estágios mais precoces. A melhora dos níveis metabólicos após o transplante hepático sugere um importante papel do Ml e da Cho no desenvolvimento da encefalopatia hepática, porém mais estudos são necessários.

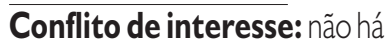

\section{SUMMARY}

Cerebral Magnetic Resonance SPECtroscopy In PATIENTS With HEPATIC ENCEPHALOPATHY: ANALYSIS BEFORE AND AFTER LIVER TRANSPLANTATION

OBIECTNES. To determine the metabolite levels (myo-inositol [MI], choline [Cho], glutamate $[\mathrm{G} / \mathrm{x}$, creatine $[\mathrm{Cr}]$ and $\mathrm{N}$-acetylaspartate [NAA7) visible on magnetic resonance spectroscopy in patients with chronic hepatic failure, before and after liver transplantation and to correlate these data with results of neuropsychiatric tests and clinical findings.

METHODS. Twenty five patients with chronic hepatic failure from the Liver Transplantation Unit of the Federal University of Parana were prospectively studied. Patients were submitted to clinical evaluation and magnetic resonance spectroscopy. Thirty healthy volunteers also submitted to the same evaluations. Sixteen of the 25 patients were evaluated after liver transplantation.

RESULTS. Before liver transplantation, significant reductions in MI/Cr and Cho/Crand a significant increase in Glx/Crwere observed in patients with hepatic encephalopathy compared with healthy subjects. The Ross's criteria for spectroscopic diagnosis of the hepatic encephalopathy (MI/Cr and Cho/Cr lower than 2 SD of controls) demonstrated a sensitivity of $61.54 \%$, specificity of $91.67 \%$ and accuracy of $76 \%$, further Cho/Cr was the best parameter. Spectroscopy after liver transplantation showed 
changes in the metabolite ratios compared with the pretransplantation status.

Conclusion. Magnetic resonance spectroscopy permits an accurate diagnosis of hepatic encephalopathy. Improvement of metabolic ratios after liver transplantation suggests an important role of Ml and Cho in the development of hepatic encephalopathy. [Rev Assoc Med Bras 2009; 55(I): 35-9]

KEY WORDS: Spectrum analysis. Brain disease. Liver transplantation. Magnetic resonance spectroscopy.

\section{REFERÊNCIAS}

I. Adams RD, Victor M, Ropper AH. Special techniques for neurologic diagnosis. In: Adams RD, Victor M, Ropper AH, editors. Principles of neurology. $6^{\text {th }}$ ed. New York: McGraw-Hill; 1997. p. 33-38.

2. Bárány M, Langer BG, Glick RP, Venkatasubramanian PN, Wilbur AC, Spigos DG. In vivo $\mathrm{H}-\mathrm{I}$ spectroscopy in humans at 1,5T. Radiology. 1988; 167:839-44.

3. Bertolucci RG, Brucki SMD, Campacci SR, Juliano Y. O mini-exame do estado mental em uma população geral. Arq Neuropsiquiatr. 1994;52:1-7.

4. Brucki SMD, Malheiros SMF, Okamoto $H$, Bertolucci PHF. Dados normativos para o teste de fluência verbal categoria animais em nosso meio. Arq Neuropsiquiatr. 1997;55:56-61.

5. Folstein MF, Folstein SE, McHugh PR. Mini-mental state: a practical method for grading the cognitive state of patients for the clinician. J Psychiat Res. 1975;12:189-98.

6. Geissler A, Lock G, Frund R, Held P, Hollerbach S, Aandus T, et al. Cerebral abnormalities in patients with cirrhosis detected by proton magnetic resonance spectroscopy and magnetic resonance imaging. Hepatology. 1997;25:48-54.

7. Gitlin N. Subclinical portal-systemic encephalopathy. Am J Gastroenterol. 1988;83:8-I।.

8. Kurhanewicz J, Bok R, Nelson SJ, Vigneron DB. Current and potential applications of clinical I3C MR Spectroscopy. J Nucl Med. 2008; I:34I-5.

9. Hockerstedt K, Kajaste S, Isoniemi H, Muuronen A, Raininko R, Seppalainen AM, et al. Tests of encephalopathy before and after liver transplantation. Transplant Proc. 1992;22:1576-8.

10. Kreis R, Farrow N, Ross BD. Diagnosis of hepatic encephalopathy by proton magnetic resonance spectroscopy. Lancet. 1990;336:635-6.

I I. Kreis R, Ross BD, Farrow NA, Ackerman Z. Metabolic disorders of the brain in chronic hepatic encephalopathy detected with $\mathrm{H}-\mathrm{I} M \mathrm{R}$ spectroscopy. Radiology. 1992;182:19-27.

I 2. Krieger S, Jauss M, Jansen O, Theilmann L, Geissler M, Krieger D. MRI findings in chronic hepatic encephalopathy depend on portossystemic shunt: results of a controlled prospective clinical investigation. J Hepatol. 1997;27: I $21-6$

13. Krieger D, Krieger S. Markers of reversible hepatic encephalopathy. Neurology. 1997;49:1 I87-8.

I 4. Laubenberger J, Häussinger D, Bayer S, Gufler H, Hennig J, Langer M. Proton Magnetic Resonance spectroscopy of the brain in symptomatic an asymptomatic patients with liver cirrhosis. Gastroenterology. 1997; | 12:1610-6.

15. Lezak MD. Neuropsychological assessment. 3 ed. New York: Oxford; 1997.
16. Luyten PR, Den Hollander JA. Observation of metabolites in the human brain by MR spectroscopy. Radiology; 1986;161:795-8.

17. Moll J, Oliveira-Souza R, Moll FT, Bramati IE, Andreiuolo PA. The cerebral correlates of set-shifting. Arq Neuropsiquiatr; 2002;60:900-5.

1 8. Näegele T, Grodd W, Viebahn R, Seeger U, Klose U, Seitz D, et al. MR imaging and 'H spectroscopy of brain metabolics in hepatic encephalopathy: time-course of renormalization after liver transplantation. Radiology. 2000;216:683-91.

19. Neuberger J, McMaster P. Liver transplantation: indications. In: Blungart LH, editor Surgery of the liver and billiary tract. Philadelphia: WB Saunders; 1994. p.180I-10.

20. Oppong KNW, Al-Mardini H, Thick M, Record CO. Oral glutamine challenge in cirrhotic patients pre e post-liver transplantation: a psychometric and analyzed EEG study. Hepatology. 1997;26:870-6.

21 . Mullen KD. Imaging may help diagnose hepatic encephalopathy. In: American Association for the Study of Liver Diseases $58^{\text {th }}$ Annual Meeting; 2007. Abstract 768.

22. Rovira A, Alonso J, Córdoba J. MR imaging findings in hepatic encephalopathy. Am J Neuroradiol. 2008;26:1936-59.

23. Rehnström S, Simert G, Hanssons JA, Johson G, Vang J. Chronic hepatic encephalopathy. A psychometrical study. Scand J Gastroenterol. | 977; | 2:305-। |.

24. Romero-Gómez M, Boza F, Garcia-Valdecasas MS, Garcia E, Aguilar-Reina J. Subclinical hepatic encephalopathy predicts the development of overt hepatic encephalopathy. Am J Gastroenterol. 2001;96:2718-23.

25. Rosa H. Encefalopatia Hepática. In: Kalil NA, Coelho J, Strauss E. Fígado e vias biliares: clínica e cirurgia. Rio de Janeiro: Revinter; $200 \mathrm{I}$. p. $102-9$.

26. Ross BD, Kreis R, Ernst T. Clinical tools for the 90s: magnetic resonance spectroscopy and metabolite imaging. Eur J Radiol. 1993; 14:128-40.

27. Ross BD, Jacbson S, Villamil F, Korula J, Kreis R, Ernst T, et al. Subclinical Hepatic Encephalopaty: proton MR spectroscopic abnormalities. Radiology. 1994; 193:457-63.

28. Miese F, Kircheis G, Wittsack HJ, Wenserki F, Hemker J, Mödder U, et al. H-MR spectroscopy magnetization transfer, and diffusionweighted imaging in alcoholic and nonalcoholic patients with cirrhosis with hepatic encephalopathy. Am J Neuroradiol. 2006;27:1019-26.

29. Weissenborn K, Ahl B, Fischer-Wasels D, Van den Hoff J, Hecker H, Burchert W, et al. Correlations between magnetic resonance spectroscopy alterations and cerebral ammonia and glucose metabolism in cirrhotic patients with and without hepatic encephalopathy. Gut. 2007;56:1736-42.

30. Thomas MA, Huda A, Guze B, Curran J, Bugbee M, Fairkanks L, et al. Cerebral 'H MR spectroscopy and neuropsychologic status of patientas with hepatic encephalopathy. AJR Am J Roentgenol. 1998; 171:112330.

31. Wechsler D. Wais-R Manual: the psychological corporation Orlando: Harcout Brave Jovanovich, Inc.; 198I.

32. Patel N, White S, Dhanjal NS, Oatridge A, Taylor-Robinson SD. Changes in brain size in hepatic encephalopathy: a coregistered MRI study. Metab Brain Dis. 2005;19:431-5.

Artigo recebido: 03/05/07

Aceito para publicaçãa: 17/08/08 